\title{
A Long Way from Transfer to Transition: Challenges for Pediatric and Adult Nephrologists
}

\author{
Johanna Lemke, M.D. ${ }^{1}$, \\ Lars Pape, M.D. ${ }^{2}$ \\ Jun Oh, M.D., Ph.D. ${ }^{1}$ \\ University Medical Center Hamburg- \\ Eppendorf', Hamburg, Medical School \\ Hannover ${ }^{2}$
}

Corresponding author: Jun Oh, M.D., Ph.D. Department of Pediatric Nephrology University Medical Center Hamburg/ Eppendorf Martinistrasse 52, 20246

Hamburg, Germany

Tel: +49-(0)40-7410-52702/51200

Fax: +49-(0)40-741055053

E-mail:j.oh@uke.de

Received: 17 March 2018

Revised: 23 March 2018

Accepted: 2 April 2018
Significant advances in the diagnosis and medical care of children with chronic kidney disease (CKD) are major reasons for the better survival rates of children and adolescents with CKD than the survival rates reported in previous decades. These patients are reaching adulthood, and therefore require a transition to adult medical care. This transition phase is well-recognized to be associated with considerably increased morbidities and medical problems, such as non-adherence, graft loss after transplantation, and loss to follow-up. Low adherence increases morbidity and medical complications and contributes to poorer qualities of life and an overuse of the health care system. However, these tragic outcomes may be avoidable through a structured and well-defined transition program. In the last decade, there has been increasing interest to resolve these medical and psychological problems that occur during the transfer of young adult patients from pediatric to adult renal units. The aims of a successful transition from pediatric to adult medical care include enhancing the individual development of better healthcompetence and stabilizing, or even improving, the state of health. This review will focus on various aspects of the transition phase of adolescents who have CKD or who underwent kidney transplantation from pediatric to adult nephrology care.

Key words: Transition, Transplantation, Chronic kidney disease (CKD), Children

\section{Introduction}

In 1984, US Surgeon General C. Everett Koop, MD, cohosted a national invitational conference focusing on the needs of adolescents and teenage children with chronic and disabling conditions ${ }^{1}$. For the first time, issues of health care transitions were on US national radar. This novel attention was brought about by significantly improved survival rates during the 1970s and early 1980s for children born with chronic and disabling conditions. Today, nearly three decades after the first conference, the process of transitioning adolescents with special health needs to adult health care appears to be notably advanced of where it was at that time. Clearly, there is more awareness nowadays of the issues regarding adolescents and young adults with chronic diseases than there were generations ago. Factors that facilitate the transition process have been more clearly established. But it has also become evident, that the medical community worldwide has yet to put into place a variety of changes to ensure that young people, most dependent on coordinated health care services are able to make the transition to the adult health care system and 
still receive the attention and services that they still deserve. These particular accounts, also for children with chronic kidney disease (CKD) and patient suffering from rare and ultra-rare diseases. There have been dramatic improvements in the survival rates of pediatric patients with CKD and after kidney transplantation. The goal for these patients is, that with the help of a purposeful and planned process in which adolescents and young adult patients assume progressively increasing responsibility for their health condition management, to maximize lifelong renal function and to optimize quality of life and survival before and after kidney transplantation.

\section{Adherence, the main barrier of the transition period}

Hynes and colleagues define the term adherence as "the extent to which a person's behavior, in terms of taking medications, following diets, or executing lifestyle changes, coincides with medical or health advice"2). Although, these two terms "compliance" and "adherence" are still often used interchangeably, adherence should be preferred because it focuses on whether a person adheres to the medical regimen rather than passively just follows doctor's orders without really understanding the origin background of these recommendations $s^{3}$. Adherence implies cooperation, partnership and understanding between patients and care takers. Medical personnel must always be alert to the increased prevalence of impaired adherence to treatment particular during teen years. Adolescents are expected to take increasing responsibility regarding their health and health care. However, adolescence is a very fragile and sensitive period due to physical, pubertal and cognitive maturation, in addition to psychosocial changes, the development of independent social relationships. During the adolescence, the child is proceeding from a complete dependency to a more or less autonomous life style ${ }^{4)}$. This leads to many problems in adherence of teenagers and young adolescents that have a negative impact on their future health.

\section{Transition in children with CKD and after kidney transplantation}

The impact of CKD on adolescents and young adults can be challenging. Transplanted patients are at very high risk for severe and irreversible complications of unsuccessful transition. Especially graft loss is most frequent at the age of 16-24 years. Cardiovascular disease is the leading cause of increased mortality and morbidity in CKD patients. Among these patients aged 15-19 years, cardiovascular event rates are nearly 1000 -fold higher than those of their age matched peers, and the mortality rate for those aged 25-34 years is similar to that of 75-year-olds in the general population ${ }^{5,6}$.

For adolescents and young adults with pediatric onset $\mathrm{CKD}$, risk factors associated with the high cardiovascular mortality rate begin early, with calcium-phosphate imbalance, chronic and acute inflammations, hypertension, fluid overload, and anemia.

Minimizing complications frequently requires close adherence to complex and restrictive, medical regimens, particularly for those on dialysis. In addition, frequent visits in the outpatient clinics and many long-term stays in the hospital with possible interventions necessary can minimize the adherence of these patients. Skipping medications and forgetting appointments, poor adherence to fluid restriction before transplantation will lead to severe and irreversible damage. The complicated lives of adolescents on dialysis are often made more challenging by the need for transition ${ }^{7}$. This has been reported for treatments of other severe chronic health conditions such as diabetes, cystic fibrosis or organ transplant possibly resulting in lifethreatening complications.

Low adherence in teenage years leads to various consequences for patients themselves, their families and the health care system. For chronic conditions, low adherence increases morbidity and medical complications (hospitalization), contributes to poorer quality of life (fatigue and absenteeism in school) and an overuse of the health care system (unnecessary medical consults and investigations) ${ }^{8)}$. Low adherence increases health care costs and may lead a health care team to withhold treatment. In the case of organ transplantation, low adherence is a leading cause of graft rejection and may cause death ${ }^{9}$. 


\section{Establishment of a successful transition}

Until now, this transition has not been uniformly established in all pediatric nephrology units worldwide. First of all, the actual transition process, a long-term controlled passage from pediatric to adult medical care, has to be differentiated from the direct transfer of medical care ${ }^{10)}$.

Various transition models are currently discussed: first, the interposition of an adolescent clinic, or even a combined continuous outpatient clinic for both adolescent and young adults including pediatric and adult nephrologists. The establishment of such a transitions or adolescent clinic as a role model for new health care structure, might help to decelerate the progression of any kidney disease in the future. National and regional training-programs, using new ITtechnology and social media, are beginning to bear fruit. In the US, training programs not only include patients, nurses and doctors, but also parents and siblings are guided through this transition phase. In Germany, there is a government and health insurance company funded transition program ("Berlin Transition program") to coordinate the procedure of transition. It also supports specific medical service (intensified communication, mobile apps etc.) that usually are not being compensated by health insurance. Initially, it was established for the transition of adolescents with type 1 diabetes and epilepsy, but since a few years, renal patients are also eligible to be included into this service. An important part of this program is a structured and welldefined pathway to regulate the transfer of pediatric patients, including transition-specific services: such as the intensification of the communication between caretakers, families and patients and the clarification of the necessity of a prepared transition. In some cases, the installation of a case-manager to secure the flow of information and to organize the numerous appointments seems reasonable. Actually a German wide randomized, prospective trial evaluating the efficacy of the Berlin Transition Program in adolescents after pediatric kidney transplantation is carried out $^{11)}$. Additionally, in Germany an educational transistion program "Endlich Erwachsen" (finally adult) exists for more than 15 years, that educates adolescents concerning disease management, self-esteem, sexuality, medications, sports and may other important subjects (www.endlich-erwachsen. de).

\section{Recommendation for the transitioning from pediatric to adult care}

So far, there are two parts of the transition process described: the first block already starts years before the actual separation process of the patients. It should begin no later than at the age of sixteen. The second block affects the time right after the first appointment at an adult clinic. However, many pediatric centers already initiate the first block even earlier than just described. These first preparations should always include the families. It should be the first and most important goal to enhance the patient's independence, the skills to organize own appointments, and his ability to communicate confident with the medical staff. One helpful tool is to provide a checklist to the patient and together log off all achieved milestones of the transition process. Periodically, these achievements should be discussed with the patient and the individual time-point for the switch of clinics should be determined together.

A successful transition can only be achieved when parents are willing to let the patient go and take over their own responsibility. This parental milestone can be particular hard to achieve, because after years of very close and intense responsibilities it is not easy to step back and let the patient make his or her own decisions. Particular helpful are specific training courses for affected parents.

This also accounts for involved doctors. Many medically complex pediatric patients have had the same medical team since they were very young; consequently, very strong bonds and trusting relationships have been established between parents and medical staff.

\section{Practical Application}

A transitions or transfer clinic should set an individual and specific algorithm for each patient. Leading physicians (and nurses) in both pediatric and adult centers should coordinate and educate on transition issues. The concept of transition should be introduced to families in early adolescence as early as 12-14 years. For a continuous transition, alternate appointments at the pediatric and adult nephrologist are essential at first. Alternatively, a specific adolescent consultation can be established in adult nephrology units 
or outpatient clinics. Here, the patient gets to know the adult nephrologist through a few combined appointments (pediatric and adult nephrologist) in the pediatric center. Further the patient will be directly transferred to the adolescent consultation. This allows teenagers and young adults to exchange experiences and information. Furthermore, it enables the physician to address the needs of the patients more specifically since taking care of this patient cohort demands time for conversation and expertise concerning age-related problems. Therefore, it is also suggested to create a network of multiple professionals, such as social workers, psychotherapists and teachers. Patient and family education about the disease and medication are additional important elements for a successful transfer process.

Designated physicians on both sides should ensure continuous monitoring of the transition process and build patient's confidence and reliance. Starting off, a 'transfer report' covering the full patient's medical history should be written by the pediatric nephrologist. This should also include physical growth, (school) education as well as social and psychological development. The starting point of transition should be agreed on commonly by all parties (patient, family, pediatric and adult nephrologist). It should take place during a time without significant medical or social problems and ideally after completing school education, especially if social support is lacking. Treatment plans by other subspecialties should be considered as well. As part of the alternating appointments the documentation and the further procedure of each appointment should be stated in a report. The state of transition should be shared with the patient appropriate to his or her intellectual development.

To recognize the patient's independence, it is essential for both pediatric and adult nephrologists to initially invite the patient without the parents, starting approximately at the age of fifteen. This allows the patient to talk about intimate topics and problems. Consequently, going through the patient's medication together and discussing current (medical) problems will help achieve a self-responsible interaction. The communication through the parents will be gradually reduced and focused towards the patient. If desired other family members or even partners should be involved. Access to group sessions or even holiday camps with other transitioning adolescents should be offered additionally.

Another important issue concerning practical aspects is the additional workload that arises from the described interventions. National health systems will have to include the additional costs for personnel in order to achieve the goals mentioned.

\section{Conclusions}

It is still a very long way from just a simple transfer to the foundation of a more sophisticated and organized transition process in the field of pediatric nephrology. However, the efforts to establish such a transition clinic are worthwhile because they increase health and quality of life for patients and their families. Though, this additional workload for physicians and their teams has to be appreciated by governments and health insurance companies all over the world.

\section{Conflicts of interest}

No potential conflict of interest relevant to this article was reported

\section{References}

1. Blum RW. Transition to adult health care: setting the stage. J Adolesc Health 1995;17:3-5.

2. Haynes RB. Introduction. In: Haynes RB, Taylor DW, Sackett DL, editors. Compliance in Health Care. Baltimore: John Hopkins University Press; 1979:1-7.

3. Lemanek KL, Kamps J, Chung NB. Empirically supported treatments in pediatric psychology: Regimen adherence. J Pediatr Psychol 2001;26:253-75.

4. Hamilton J, Daneman D. Deteriorating diabetes control during adolescence: Physiological or psychosocial. J Pediatr Endocrinol Metab 2002;15:115-26.

5. Oh J, Wunsch R., Turzer M., Bahner M, Raggi P, Querfeld U, et al. Advanced coronary and carotid arteriopathy in young adults with childhood-onset chronic renal failure. Circulation 2002;106 (1):100-5.

6. Mitsnefes MM, Cardiovascular complications of pediatric chronic kidney disease. Pediatr Nephrol 2008;23(1):27-39. 


\section{www.chikd.org}

7. Kane S, Huo D, Aikens J, Hanauer S. Medication nonadherence and the outcomes of patients with quiescent ulcerative colitis. Am J Med 2003;114:39-43.

8. Lemanek KL, Kamps J, Chung NB. Empirically supported treatments in pediatric psychology: Regimen adherence. J Pediatr Psychol 2001;26:253-75.

9. Falkenstein K, Flynn L, Kirkpatrick B, Casa-Melley A, Dunn S. Noncompliance in children post-liver transplant: Who are the culprits? Pediatr Transplant 2004;8:233-6.
Lemke J, et al. • Transition Phase in Pediatric Renal Patients 11

10. Prüfe J, Dierks ML, Bethe D, Oldhafer M, Müther S, Thumfart J, et al. Transition structures and timing of transfer from paediatric to adult-based care after kidney transplantation in Germany: a qualitative study. BMJ Open 2017;7(6):e015593.

11. Kreuzer M, Prüfe J, Bethe D, Vogel C, Großhenning A, Koch A, et al. The TRANSNephro-study examining a new transition model for post-kidney transplant adolescents and an analysis of the present health care: study protocol for a randomized controlled trial. Trials 2014;15:505 doi:10.1186/1745-6215-15-505. 